\title{
REVIEW \\ Shedding the load of hypertension: The proteolytic processing of angiotensin-converting enzyme
}

\author{
Mario R W Ehlers, Kerry Gordon, Sylva L U Schwager, Edward D Sturrock
}

A number of membrane proteins are enzymatically cleaved or 'shed' from the cell surface, resulting in the modulation of biological events and opening novel pharmaceutical approaches to diverse diseases by targeting shedding. Our focus has been on understanding the shedding of angiotensin-converting enzyme (ACE), an enzyme that plays a pivotal role in blood pressure regulation. The identification of novel hereditary ACE mutations that result in increased ACE shedding has advanced our understanding of the role of ACE shedding in health and disease. Extensive biochemical and molecular analysis has helped to elucidate the mechanism of ACE shedding. These findings point to the potential therapeutic role of targeting shedding in regulating tissue ACE levels in cardiovascular disease.

S Afr Med J 2012;102(6):461-464.

\section{Shedding of membrane proteins}

Barely 20 years have elapsed since our first description of membrane protein shedding as a general phenomenon in biology. ${ }^{1}$ Also described as 'membrane protein solubilisation' but now generally referred to as 'ectodomain shedding', this is a process whereby proteins anchored in the cell membrane are cleaved and released by specialised proteases called 'sheddases'. It is now estimated that up to $10 \%$ of all membrane proteins on somatic cells are processed in this way. ${ }^{2}$

Why is ectodomain shedding so widespread? Diverse types of membrane proteins are involved, most commonly adhesion proteins, various kinds of receptors, and cytokines and enzymes tethered to the cell surface. Ectodomain shedding allows for the rapid modulation of biological events on cell surfaces, especially cell-cell communication. In many cases, cell activation in response to endocrine or immune signals requires changes in cell-surface expression of receptors, adhesion molecules or cytokines, or the production of soluble forms of these proteins, and this is rapidly achieved by shedding. ${ }^{1}$ Many other physiological processes, from neural development to regulation of cell growth, also rely on shedding.

Given this ubiquity, it is not surprising that genetic disruption of one of the principal sheddases, ADAM17, is lethal during embryogenesis. ${ }^{2}$ We now know that there are many sheddases,

Mario Ehlers is the Deputy Director of the Clinical Trials Group at the Immune Tolerance Network, USA. He was formerly Professor and Head of the Department of Medical Biochemistry at the University of Cape Town (UCT). His principal therapeutic areas of interest include diabetes, cardiovascular disease, and autoimmunity. Edward Sturrock is Professor of Medical Biochemistry at UCT and an author of more than 75 peer-reviewed scientific papers. His research interests include structure-function studies of metalloproteases and the development of next-generation ACE inhibitors for treating hypertension and fibrosis. Sylva Schwager is a protein biochemist and co-author of more than 30 scientific papers. She obtained her undergraduate and postgraduate degrees from UCT. Her research interests include the ectodomain shedding of membrane proteins, the structure and function of metalloproteases and drug discovery and development. Kerry Gordon is a postdoctoral fellow in the Department of Molecular and Cell Biology at UCT. Her research interests include the role of envelope glycosylation in HIV and proteinprotein interactions and ectodomain shedding of ACE.

Corresponding authors: M Ehlers (mehlers@immunetolerance.org) and E Sturrock (edward.sturrock@uct.ac.za) although the most important appear to fall into 2 classes, both of which are zinc-dependent proteases: ADAM (a disintegrin and metalloproteinase) proteases, such as ADAM17 and ADAM10, and MMPs (matrix metalloproteases) (Fig. 1). There is significant interest in finding specific sheddase inhibitors, because of enormous pharmaceutical potential in diverse diseases, including autoimmunity, cancer and Alzheimer's disease.

\section{The discovery of ACE shedding}

Among membrane proteins that are shed, a significant proportion are cell surface enzymes, often called ecto-enzymes, including angiotensin-converting enzyme (ACE), which we were the first to show was spontaneously shed in a mammalian cell line. ${ }^{3}$ ACE is a large zinc-dependent protease that is a key component of the reninangiotensin system (RAS); ACE inhibitors are important drugs for the treatment of heart failure, hypertension, myocardial infarction, and diabetic nephropathy. ${ }^{4} \mathrm{ACE}$ is widely expressed in endothelial and epithelial cells, where it is anchored in the cell membrane by a lipid-soluble domain near the C-terminal end of the protein. The enzyme can be viewed as having the shape of a lollipop: the bulk of the protein forms the globular part, which is connected via a stalk to the membrane anchor (Fig. 2). It is widely believed that it is the membrane-bound ('tissue') ACE that is principally responsible for its role within the RAS, although ACE was originally discovered as a soluble protein in plasma.

Soluble ACE is found in plasma, seminal fluid, cerebrospinal fluid and urine. Plasma ACE levels increase in certain inflammatory conditions, notably sarcoidosis, and it is believed the source is activated monocytes and macrophages. ${ }^{5}$ Elevated plasma ACE levels have also been linked to ACE gene polymorphisms, in some cases leading to very high plasma ACE levels. However, the role of soluble ACE in plasma is unclear. In terms of blood pressure regulation, studies in humans and in animals, including transgenic and knockout models, have shown that only the tissue-bound form is necessary and sufficient. ${ }^{6}$ It is more plausible that soluble ACE in plasma reflects a process whereby levels of tissue-bound ACE are being fine-tuned, thereby regulating local tissue RAS. If this is true, then soluble ACE is merely a byproduct of RAS regulation at the tissue level.

Despite the absence of definitive data, it is probable that soluble ACE has subtle biological functions in its own right. For example, soluble ACE in plasma offers a means for delivery of the enzyme to tissues that do not express their own ACE, thereby enabling local angiotensin II production with regional effects on vascular 


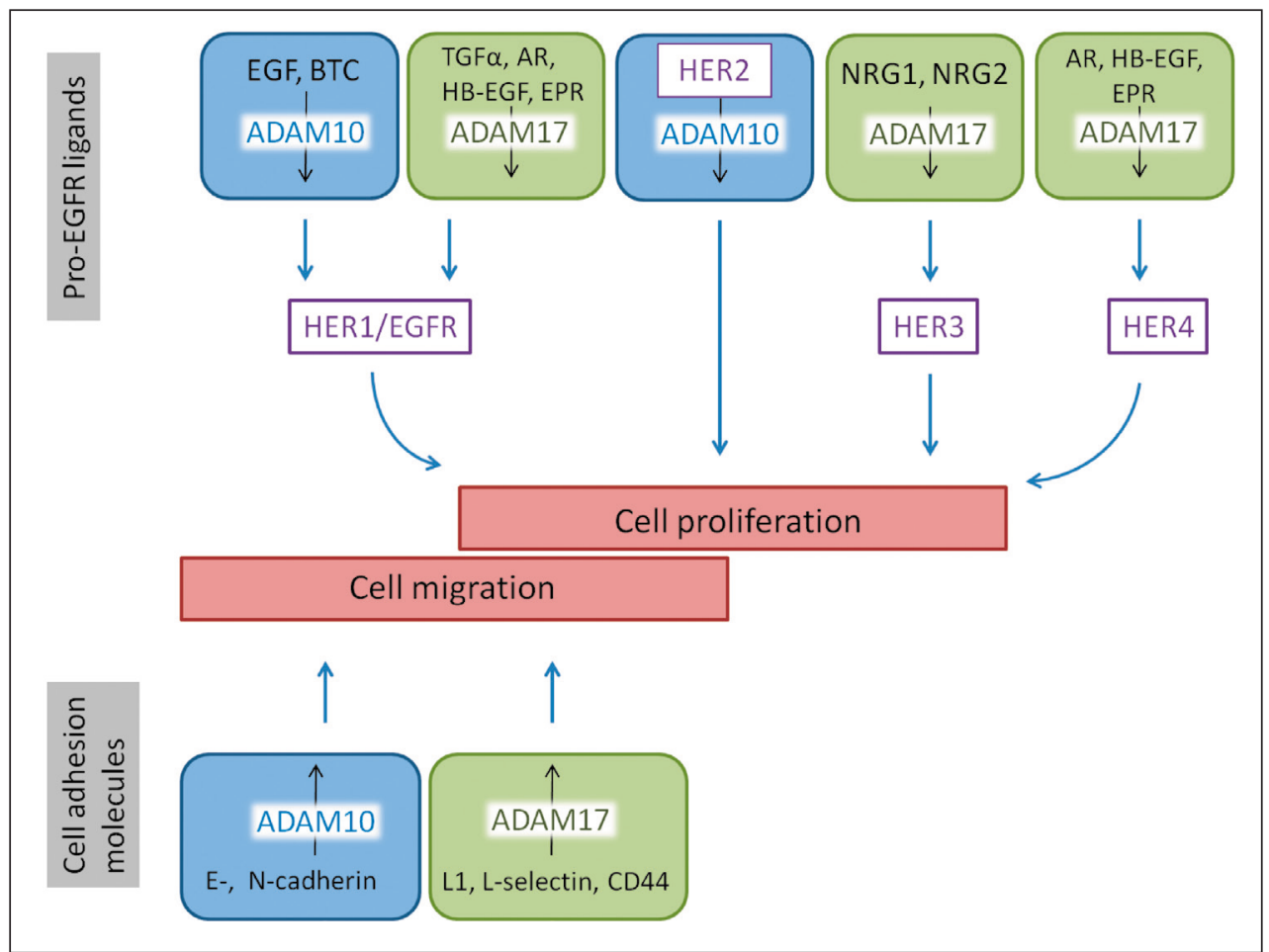

Fig. 1. ADAM10 and ADAM17 are sheddases that play important roles in the regulation of cell migration and proliferation in cancer. Tumour cell migration and invasion are increased by ADAM10- and ADAM17-mediated shedding of cell adhesion molecules. Both sheddases also increase tumour proliferation due to the release of pro-EGFR ligands and EGFR transactivation. The figure was adapted from Saftig and Reiss. ${ }^{19}$

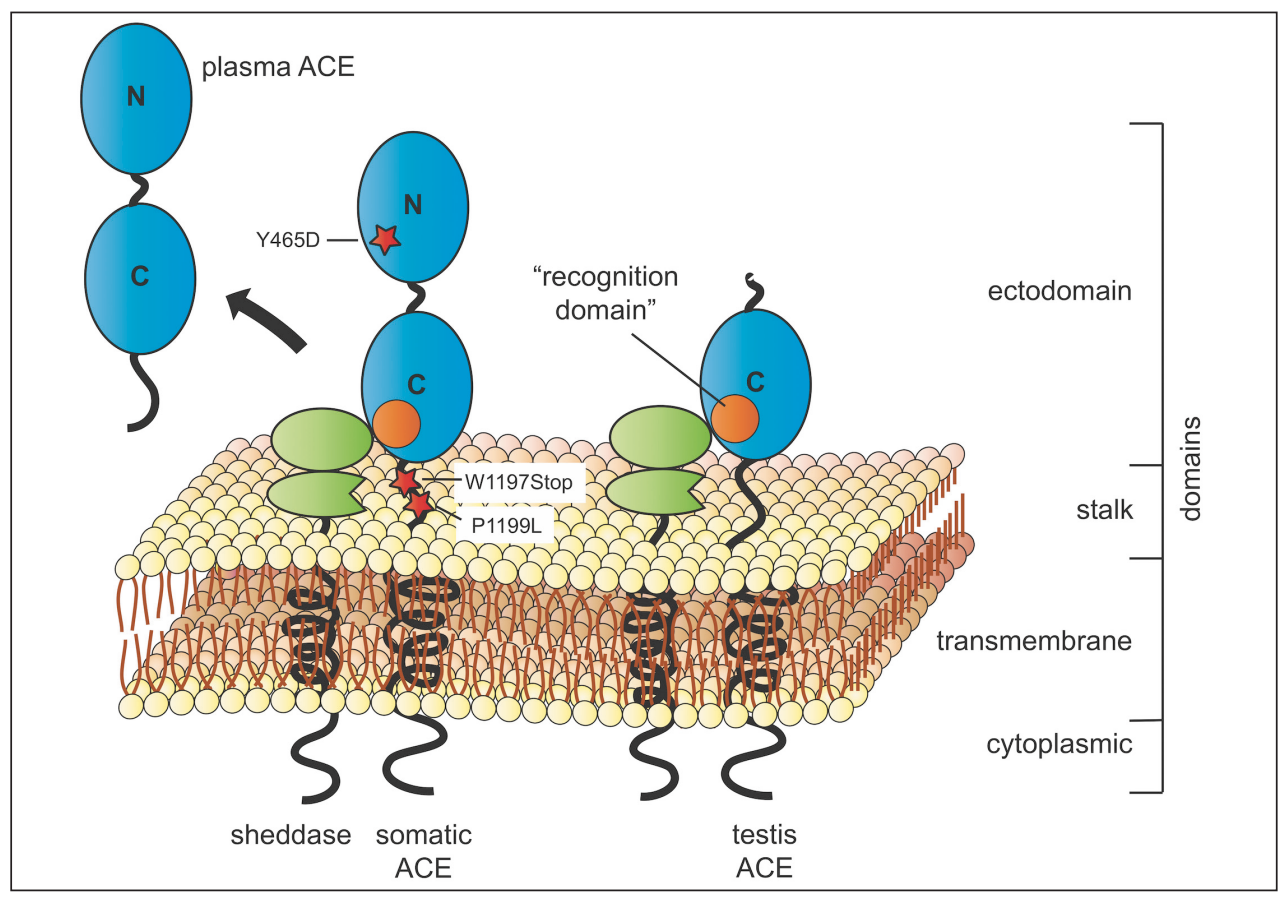

Fig. 2. Schematic representation of the ectodomain shedding of somatic and testis ACE isoforms. ACE domain structure is described in Fig. 3. The transmembrane domain (black coil) spans the lipid bilayer of the cell membrane. Cleavage of the stalk by the sheddase (green) leads to shedding. ACE gene mutations (red stars) P1199L, W1197Stop, and Y465D result in enhanced shedding and increased levels of soluble ACE in plasma.

tone and fluid and electrolyte homeostasis. Similarly, monocytes and macrophages expressing ACE may require both membranebound and soluble forms for immune activation and regulation, as has been shown for other immune system proteins, such as TNFa and the receptors for $\mathrm{TNF} \alpha$ and IL- $6 .^{2}$ In the male genital tract, a special isoform of ACE is expressed on the cell membrane of spermatozoa, but is later shed at a specific stage of spermatozoan maturation and appears in the seminal fluid. Again, the precise roles of the membrane-bound and soluble forms of the enzyme are poorly understood, but genetic disruption of germinal ACE in rodents leads to male infertility. ${ }^{6}$

\section{The mechanism of ACE shedding}

In our laboratory we have focused on the mechanism of ACE shedding: how does the sheddase recognise its target; where in the target protein does the cleavage occur; how is the process regulated; and what are the identities of the sheddases? As shown in Fig. 2, we now know that membrane proteins that are the targets of sheddases generally have a stalk that connects the ectodomain to the membrane anchor. This stalk is open and accessible and contains the cleavage site. This has led to the notion that sheddases function like lawnmowers that move over the surface of the cell and cut any protein stalks in their path. Although this is true in broad outline, our research indicates that sheddases follow certain rules: the sheddase not only looks for an open stalk but positions itself relative to the ectodomain and requires a minimum distance from the membrane. ${ }^{7}$ Even more intriguing is evidence that the sheddase binds to a 'recognition domain' in the target protein before cleaving the stalk.

Many of these concepts emerged from our work on ACE, in which we have the advantage of a natural experiment. As shown in Fig. 3, there are in fact 2 kinds of ACE: the common form of the enzyme that is widely distributed ('somatic ACE') and an ACE variant, first cloned in our laboratory, which is only expressed in spermatozoa ('testis ACE'). Somatic ACE contains 2 similar but distinct domains arranged in tandem: the second of the 2 domains (the $\mathrm{C}$ domain) is identical to 


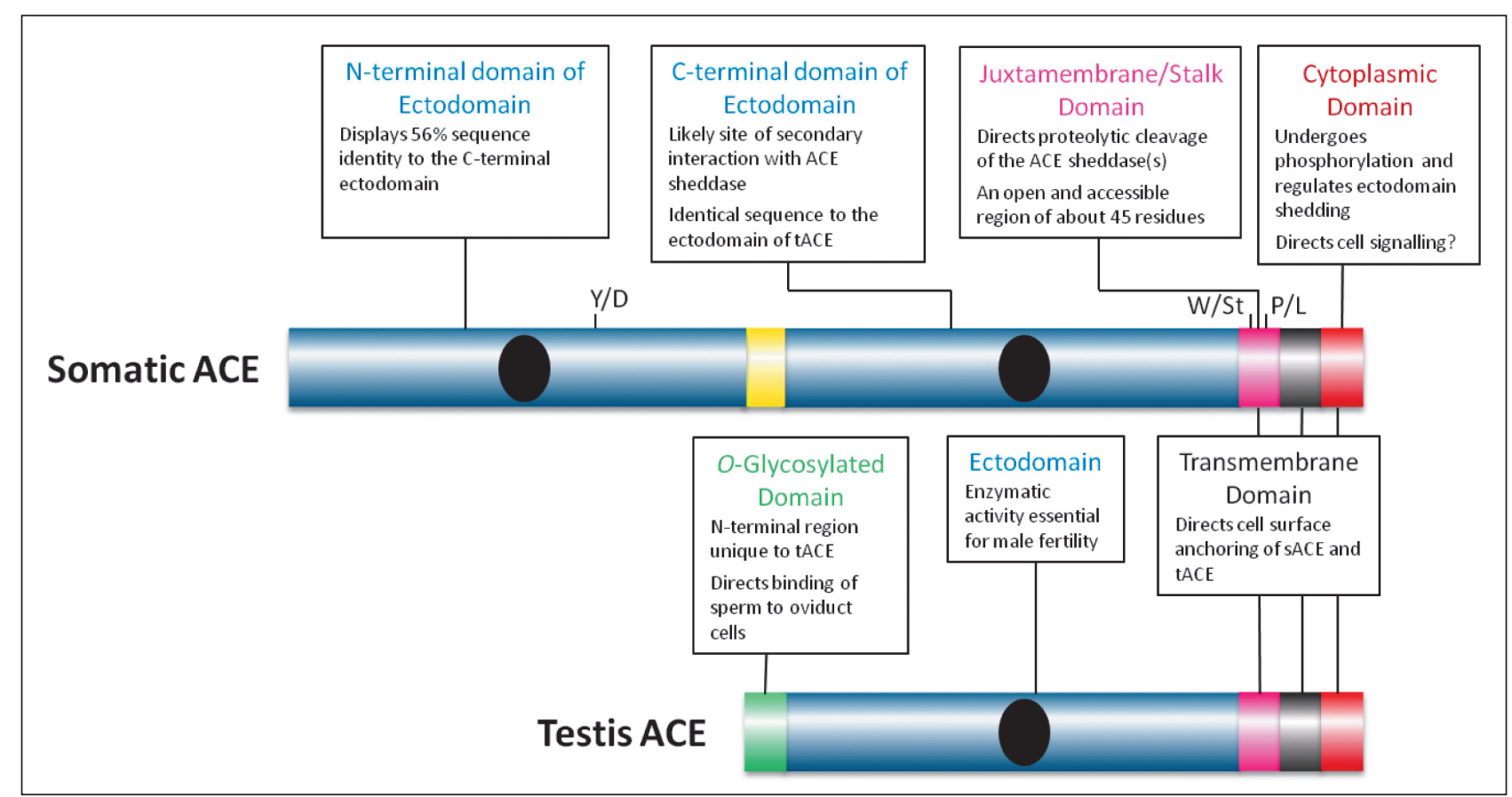

Fig. 3. The structural organisation of somatic and testis ACE proteins. The coloured bars are a schematic illustration of the organisation of the core protein domains. The 3 mutations found in patients that affect ectodomain shedding are indicated using the single-letter amino acid code and St for stop codon (see also Fig. 2). The black ovals denote the active sites with their characteristic zinc-binding motifs. The yellow box denotes a short unstructured linker region of approximately 14 residues that joins the $N$ and $C$ domains of somatic ACE.

the bulk of the testis enzyme and both proteins share identical stalk, membrane, and cytoplasmic domains (Fig. 3). Despite this, shedding of testis ACE is much more efficient than shedding of somatic ACE. Why? Work from our and other laboratories has indicated that this is most likely because there is a sheddase recognition domain in the $\mathrm{C}$ domain of ACE, which in somatic ACE is partly occluded by the presence of the second domain ( $\mathrm{N}$ domain), ${ }^{8,9}$ Indeed, when a mutant ACE was constructed in which the N domain of somatic ACE was fused directly to the stalk, this mutant was not shed, suggesting that it lacked a critical recognition element for the sheddase. ${ }^{9}$

\section{Candidate ACE sheddases}

The search for the ACE sheddase is ongoing. The 2 sheddases that are most widely implicated in shedding diverse membrane proteins - ADAM17 and ADAM10 - are not involved in ACE shedding. ${ }^{10}$ The difficulty in identifying the ACE sheddase is partly because there are several alternate pathways and sheddases that contribute to this process. Interestingly, the ACE sheddase appears to be very similar (but not identical) to the so-called ' $\alpha$-secretase', a sheddase that cleaves the amyloid precursor protein (APP), a protein that is implicated in the pathogenesis of Alzheimer's disease. Although there does not appear to be a connection between ACE and APP, this similarity highlights the range of physiological and pathological processes in which shedding plays a role.

\section{ACE mutations that affect ectodomain shedding and their implications}

Recently, 3 novel ACE gene polymorphisms have been described that are associated with increased plasma ACE levels (see Figs 2 and 3).

First, a mutation was identified in subjects from studies of unrelated individuals in Europe. ${ }^{11}$ Patients with elevated plasma ACE had a single amino acid change in the stalk region (Pro1199Leu). Expression of recombinant ACE with this mutation in mammalian cells $^{11}$ caused increased shedding of ACE, most likely because of a conformational change in the stalk allowing better access by the sheddase.

A second mutation in a family of African-Americans (W1197Stop) ${ }^{12}$ results in a truncated ACE protein that lacks the membrane and cytoplasmic domains, leading to the direct production of soluble ACE and explaining the elevated plasma levels. The W1197Stop and Pro1199Leu mutations do not produce any apparent clinical pathology.

Collaboration of our group with the University of Chicago has revealed a third ACE mutation present in a family of individuals where the affected members suffer from intermittent nausea, vomiting, fatigue and depression. ${ }^{13}$ Investigation revealed dramatically elevated levels of plasma ACE (approximately 10-fold higher than normal) associated with a mutation in the ACE ectodomain (Y465D). Expression of recombinant ACE with this mutation in mammalian cells confirmed that the Y465D mutation causes increased ACE shedding. ${ }^{13}$ This is remarkable because the mutation is distant from the stalk cleavage site and probably causes a change in the relative orientation of the two domains, allowing better access of the sheddase.

The Y465D mutation is the first ACE polymorphism described that is associated with clinical pathology. Dysregulation of ACE expression and processing may produce the pathology by altering plasma levels of vaso- and neuropeptides degraded by ACE. A likely candidate is substance $\mathrm{P}$, a peptide involved in pain and chronic inflammation, elevated plasma levels of which are associated with nausea, vomiting, depression and fatigue. ${ }^{14} \mathrm{ACE}$ is one of the major enzymes involved in degradation of substance $\mathrm{P}^{15}$ and symptomatic individuals with the Y465D mutation were shown to have elevated plasma substance P levels. ${ }^{13}$

\section{ACE sheddases as therapeutic targets}

The dynamics between the membrane-bound and soluble forms of ACE in the local and systemic renin-angiotensin systems are complex 
and a better understanding of the role played by the ACE sheddase(s) is still required. Moreover, unlike the systemic RAS, where renin secretion by the kidney plays an important regulatory role in the processing of angiotensinogen, it is not clear how the production of angiotensin II by the tissue RAS is controlled. In terms of therapeutic interventions, an important question is whether limiting the production of angiotensin II is best achieved by blocking the activity of ACE or by inhibiting its release from the cell membrane. While circulating ACE levels might have some clinical implications in certain diseases such as metabolic syndrome ${ }^{16}$ tissue ACE is likely to be of more clinical significance in major cardiovascular diseases (CVD).$^{17}$ In general, blocking ACE activity by the use of conventional ACE inhibitors is likely to be most productive in mitigating CVD. However, inhibiting ACE shedding may offer novel therapeutic opportunities in situations in which increased local levels of angiotensin II, or other vaso- and neuropeptides, are useful. This concept of fine regulation of ACE activity at the tissue level is also being explored by an independent avenue of research in our laboratory - focused on specifically inhibiting single domains of somatic ACE, either the $\mathrm{N}$ or the $\mathrm{C}$ domain (current ACE inhibitors do not discriminate between the 2 domains). Recent studies have explored the different physiological roles of the $\mathrm{N}$ and $\mathrm{C}$ domain-active sites of ACE and have shown that inhibition of a single domain may be advantageous for targeting certain diseases, such as hypertension (C domain) and organ fibrosis ( $\mathrm{N}$ domain), or for improving the side-effect profile of current non-selective ACE inhibitors. ${ }^{18}$

\section{Conclusions}

Shedding of membrane-bound ACE occurs in all mammalian species in which this has been studied and likely plays a critical role in regulating this enzyme at the cell and tissue levels. A more detailed picture of the process of ACE ectodomain shedding will not only further our understanding of the biology of ACE but also holds promise for novel therapeutic interventions. Beyond the shedding of ACE, sheddases affect a wide and emerging range of pathologies and thus are attractive targets for future therapeutic approaches. However, the number of target proteins and tissue distribution for each sheddase varies considerably, requiring care in selectively inhibiting a sheddase in an organ associated with a specific disease without affecting the function of the protease in other tissues. Despite this, there is optimism that sheddase inhibitors may be useful in autoimmune diseases, malignancies, and neurodegenerative conditions.

1. Ehlers MR, Riordan JF. Membrane proteins with soluble counterparts: role of proteolysis in the release of transmembrane proteins. Biochemistry 1991;30(42):10065-10074.

2. Scheller J, Chalaris A, Garbers C, Rose-John S. ADAM17: a molecular switch to control inflammation and tissue regeneration. Trends Immunol 2011;32(8):380-387.
and

3. Ehlers MR, Chen YN, Riordan JF. Spontaneous solubilization of membrane-bound human testis angiotensin-converting enzyme expressed in Chinese hamster ovary cells. Proc Natl Acad Sci USA
a 1991;88(3):1009-1013

4. Acharya KR, Sturrock ED, Riordan JF, Ehlers MR. ACE revisited: a new target for structure-based drug design. Nat Rev Drug Discov 2003;2(11):891-902

5. Ehlers MR, Riordan JF. Angiotensin-converting enzyme: new concepts concerning its biological role. Biochemistry 1989;28(13):5311-5318.

6. Shen XZ, Xiao HD, Li P, et al. New insights into the role of angiotensin-converting enzyme obtained from the analysis of genetically modified mice. J Mol Med (Berl) 2008;86(6):679-684.

7. Ehlers MR, Schwager SL, Scholle RR, Manji GA, Brandt WF, Riordan JF. Proteolytic release of membrane-bound angiotensin-converting enzyme: role of the juxtamembrane stalk sequence. Biochemistry 1996;35(29):9549-9559.

8. Beldent V, Michaud A, Bonnefoy C, Chauvet MT, Corvol P. Cell surface localization of proteolysis of human endothelial angiotensin I-converting enzyme. Effect of the amino-terminal domain in the solubilization process. J Biol Chem 1995;270(48):28962-28969.

9. Woodman ZL, Schwager SL, Redelinghuys P, Carmona AK, Ehlers MR, Sturrock ED. The N domain . Woodman $\mathrm{ZL}$, Schwager $\mathrm{SL}$, Redelinghuys $\mathrm{P}$, Carmona AK, Ehlers $\mathrm{MR}$, Sturrock ED. The N domain
of somatic angiotensin-converting enzyme negatively regulates ectodomain shedding and catalytic of somatic angiotensin-converting enzyme
activity. Biochem J 2005;389(Pt 3):739-744.

10. Allinson TM, Parkin ET, Condon TP, et al. The role of ADAM10 and ADAM17 in the ectodomain shedding of angiotensin converting enzyme and the amyloid precursor protein. Eur J Biochem 2004;271(12):2539-2547

11. Eyries M, Michaud A, Deinum J, et al. Increased shedding of angiotensin-converting enzyme by a mutation identified in the stalk region. J Biol Chem 2001;276(8):5525-5532.

12. Nesterovitch AB, Hogarth KD, Adarichev VA, et al. Angiotensin I-converting enzyme mutation (Trp1197Stop) causes a dramatic increase in blood ACE. PLoS One 2009;4(12):e8282.

13. Danilov SM, Gordon K, Nesterovitch AB, et al. An angiotensin I-converting enzyme mutation (Y465D) causes a dramatic increase in blood ACE via accelerated ACE shedding. PLoS One 2011;6(10):e25952. 4. Harrison S, Geppetti P. Substance p. Int J Biochem Cell Biol 2001;33(6):555-576.

15. Ehlers MRW, Riordan JF. Angiotensin-converting enzyme: zinc and inhibitor-binding stoichiometries of the somatic and testis isozymes. Biochemistry 1991;30:7118-7126.

16. Nagi DK, Foy CA, Mohamed-Ali V, Yudkin JS, Grant PJ, Knowler WC. Angiotensin-1-converting enzyme (ACE) gene polymorphism, plasma ACE levels, and their association with the metabolic enzyme (ACE) gene polymorphism, plasma ACE levels, and their association with the metabolic
syndrome and electrocardiographic coronary artery disease in Pima Indians. Metabolism syndrome and electo

17. Dzau VJ, Bernstein K, Celermajer D, et al. Pathophysiologic and therapeutic importance of tissue ACE: a consensus report. Cardiovasc Drugs Ther 2002;16(2):149-160

18. Douglas RG, Ehlers MRW, Sturrock ED. Vasopeptidase inhibition - solving the cardiovascular puzzle? Drugs of the Future 2011;36(1):33-43

19. Saftig P, Reiss K. The 'A disintegrin and metalloproteases' ADAM10 and ADAM17: novel drug targets with therapeutic potential? Eur J Cell Biol 2011;90(6-7):527-535

Accepted 31 January 2012. 